\title{
A TOPOLOGICAL RESOLUTION THEOREM
}

\section{BY SELMAN AKBULUT AND LARRY TAYLOR}

We prove a topological analogue of the resolution theorem for algebraic varieties [H]. We show that every compact P.L. manifold $M$ admits a framed stratification (every stratum has a product neighborhood) such that after a sequence of topological blow ups performed along the closed smooth strata we get a compact smooth manifold $\widetilde{M}(\partial \widetilde{M}=\varnothing$ if $\partial M=\varnothing)$ and a degree one map (with $Z / 2$ coefficients) $\pi: \widetilde{M} \rightarrow M$. The map $\pi$ is a P.L. homeomorphism in the complement of a union of smooth submanifolds of the form $N_{i} \times W_{i}$, such that $\pi$ collapses $N_{i} \times W_{i}$ to $N_{i}$ in some order. This structure can be used to show that every compact P.L. manifold is P.L. homeomorphic to a real algebraic variety $[\mathbf{A K}]$. This also gives a nice way of defining differential forms on P.L. manifolds by pushing down the relative forms from the smooth resolution spaces.

Define an $A_{0}$-structure on a P.L. manifold to be a smooth structure, and call such manifold an $A_{0}$-manifold. Inductively define an $A_{k}$-structure on a P.L. manifold $M$ to be a decomposition

$$
M=M_{0} \cup_{\phi} \coprod_{i=1}^{r} N_{i} \times \operatorname{cone}\left(\Sigma_{i}\right)
$$

for some $r$, where $M_{0}$ is an $A_{k-1}$-manifold with boundary; each $\Sigma_{i}$ is a boundary of a compact $A_{k-1}$-manifold and is P.L. homeomorphic to a P.L. sphere; and $N_{i}$ are smooth manifolds. Finally $\phi=\left\{\phi_{i}\right\}$ are maps describing the identification (as stratified sets) $\phi_{i}: N_{i} \times \Sigma_{i} \rightarrow \partial M_{0}$ where the union is taken. We say $M$ has an $A$-structure if it has an $A_{k}$-structure for some $k$.

To describe the blowing up process, let $M$ be an $A_{k}$-manifold. Then $M=$ $M_{0} \cup \amalg_{i} N_{i} \times \operatorname{cone}\left(\Sigma_{i}\right)$ and we can choose compact $A_{k-1}$-manifolds $W_{i}$ with $\partial W_{i}=\Sigma_{i}$. Construct the obvious $A_{k-1}$-manifold $\widetilde{M}_{k-1}=M_{0} \cup \amalg_{i} N_{i} \times W_{i}$. There is the obvious P.L. map $\pi: \widetilde{M}_{k-1} \rightarrow M$ which is the identity on $M_{0}$ and collapses each $N_{i} \times W_{i}$ onto $N_{i}$. We can iterate this process to get a resolution sequence

$$
\widetilde{M}=\widetilde{M}_{0} \stackrel{\pi}{\rightarrow} \widetilde{M}_{1} \stackrel{\pi}{\rightarrow} \cdots \stackrel{\pi}{\rightarrow} \widetilde{M}_{k-1} \stackrel{\pi}{\rightarrow} M
$$

Received by the editors September 4, 1979.

AMS (MOS) subject classifications (1970). Primary 57C2 5, 55E15; Secondary $55 \mathrm{~F} 40$.

Key words and phrases. P.L. mainfolds, $A$-manifolds, stratifications, resolutions, thickenings, classifying spaces. 
$\widetilde{M}$ is smooth and clearly the composition map $\pi: \widetilde{M} \rightarrow M$ collapses $N_{i} \times W_{i}$ onto $N_{i}$.

Following [L] and [W] we define $A$-thickenings; the classifying space $B_{A}$; and the natural map $B_{A} \rightarrow B_{P L}$. Then we prove the usual structure theorem: Namely that a compact P.L. manifold $M$ has an $A$-structure if and only if the normal bundle map (thickening map)

$$
M \stackrel{\nu_{M}}{\rightarrow} B_{\mathrm{PL}}
$$

lifts to $B_{A}$. Let $P L / A$ be the homotopy theoretical fibre of $B_{A} \rightarrow B_{P L}$.

THEOREM. $B_{A} \simeq B_{P L} \times P L / A$, and $P L / A$ is the product of EilenbergMc Laine spaces $K(Z / 2, n)$ 's. The number $\delta_{n}$ of $K(Z / 2, n)$ for each $n$ in this product is given to be

$$
\delta_{n}=\left\{\begin{array}{l}
0 \quad \text { if } n<8 \\
26 \quad \text { if } n=8 \\
\text { infinite but countable if } n>8 .
\end{array}\right.
$$

Corollary. Every compact P.L. manifold $M$ has an A-structure and number of different $A$-structures (up to A-concordance) on $M$ is given by $\bigoplus_{n \geqslant 8} H^{n}\left(M: \pi_{n}(P L / A)\right)$.

Roughly an $A$ structure on $M$ gives a topological resolution on $M$ and $\bigoplus_{n} H^{n}\left(M: \pi_{n}(P L / A)\right)$ classifies different ways of resolving $M$.

OUtLINE OF PROOF. While constructing $B_{A}$ we also classify $A_{k}$-thickenings and prove the usual classification theorem for them. We then proceed to analize $B_{A_{k}}$, and $B_{A}=\lim _{k \rightarrow \infty} B_{A_{k}}$. It is standard that $\pi_{i}\left(P L / A_{k}\right)$ coincides with concordance classes of $A_{k}$-structures on $S^{i}$. Since $\pi_{i}(P L / A)=$ $\lim _{k \rightarrow \infty} \pi_{i}\left(P L / A_{k}\right)$ it follows from definitions that $\pi_{i}(P L / A)$ maps monically to the $i$-dimensional unoriented $A$ bordism group $\eta_{i}^{A}$. Next we construct a Thom spectrum, $M A$, with $\pi_{i}(M A) \approx \eta_{i}^{A}$.

Since it is clear that an $A_{k}$-manifold crossed with a smooth manifold is an $A_{k}$-manifold, we can show that $M A$ is an $M O$ module spectrum and it is now a formality that the map $\eta_{i}^{A} \rightarrow H_{i}\left(B_{A} ; Z / 2\right)$ given by

$$
\left\{M \stackrel{\nu_{M}}{\longrightarrow} B_{A}\right\} \sim\left(\nu_{M}\right) *[M]
$$

is monic. Hence $\pi_{*}(P L / A) \rightarrow H_{*}\left(B_{A} ; Z / 2\right)$ is monic, hence split. It is now easy to show that $P L / A$ is a product of $K(Z / 2, n)$ 's and to construct a map $B_{A} \rightarrow$ $P L / A$ splitting the inclusion $P L / A \rightarrow B_{A}$.

To compute $\delta_{n}$ is not so hard. To construct $B_{A}$ one proves along the way that $\delta_{n}$ is countable. For $n \leqslant 8$ one can explicitly see all the $A$-spheres so there is no problem. Above 8 things are complicated but it is not too hard to construct 
an infinite number of concordance classes in each dimension. The details will appear elsewhere $[\mathbf{A T}]$.

\section{REFERENCES}

[AK] S. Akbulut and H. C. King, Real algebraic structures on topological spaces, Bulletin Amer. Math. Soc. (N. S.) 2 (1980),

[AT] S. Akbulut and L. Taylor, A topological resolution theorem, Bulletin Amer. Math. Soc. (N. S.) 2 (1980),

[H] H. Hironaka, Resolution of singularities of an algebraic variety over a field of characteristic zero, Ann of Math. 79 (1964), 109-326.

[L] N. Levitt, Exotic singular structures on spheres, Trans. Amer. Math. Soc. 205 (1975), 371-383.

[W] C. T. C. Wall, Classification problems in differential topology-IV, thickenings, Topology 5 (1966), 73-94.

DEPARTMENT OF MATHEMATICS, RUTGERS UNIVERSITY, NEW BRUNSWICK, NEW JERSEY 08903

DEPARTMENT OF MATHEMATICS, UNIVERSITY OF NOTRE DAME, NOTRE DAME, INDIANA 46556 